\title{
Panorama prospectivo das demências no Brasil: um enfoque demográfico
}

\author{
A perspective overview of dementia in Brazil: \\ a demographic approach
}

Claudia Burlá ${ }^{1}$

Ana Amélia Camarano ${ }^{2}$

Solange Kanso ${ }^{2}$

Daniele Fernandes ${ }^{2}$

Rui Nunes ${ }^{1}$
${ }^{1}$ Faculdade de Medicina da Universidade do Porto. Alameda Professor Hernâni Monteiro 4200/319, Asprela. Porto Portugal. cburla@terra.com.br

${ }^{2}$ Instituto de Pesquisa Econômica Aplicada (IPEA).
Abstract It is only to be expected that the elderly population manifests a high prevalence of chronic diseases that jeopardize their autonomy. One example is syndromes of dementia. The scope of this paper is to make projections of the number of elderly individuals with dementia. An estimate of the current number is made by applying age-related prevalence data to the population of 65 and over, by age-group, with the figures from the Brazilian Demographic Census of 2010. The data were obtained from assessments found in the literature. For a national measure, a weighted average of the estimated prevalence was used. Prevalence rates by age and gender were calculated for the elderly Brazilian population. Current investigations show a steep increase of dementia with age. Women and illiterate individuals have a higher prevalence. The mean prevalence in Brazil is higher than that found in the rest of the world. Projections for the Brazilian population point to a small increase in the prevalence of dementia in the $65+$ age-group, from $7.6 \%$ to $7.9 \%$ between 2010 and 2020, which means 55,000 new cases a year. Dementia is a public health issue, and elicits a strong bioethical concern with aging, due to the loss of autonomy of the affected individuals and the responsibility of the health system to provide adequate care.

Key words Dementia, Ageing, Demography, Bioethics
Resumo É típico da população idosa a prevalência elevada de doenças crônicas, que comprometem a sua autonomia. Um exemplo são as síndromes demenciais. O objetivo deste trabalho é projetar o número de idosos demenciados. Para isto estimou-se o número atual, aplicando medidas de prevalência por grupos de idade à população de 65 anos e mais, enumerada pelo Censo Demográfico de 2010. Estas medidas foram obtidas nos levantamentos encontrados na literatura. Para se obter uma medida nacional, utilizou-se uma média ponderada das prevalências estimadas. Foram obtidas taxas de prevalência por idade e sexo para a população idosa brasileira. Pesquisas existentes mostram que a demência cresce acentuadamente com a idade. As mulheres e os analfabetos apresentam uma prevalência mais elevada. A prevalência brasileira média apresenta-se mais alta que a mundial. Projeções para a população brasileira apontam para um pequeno crescimento na taxa de prevalência de demência na população com 65 anos e mais, de 7,6\% para 7,9\% entre 2010 e 2020, ou seja, 55.000 novos casos por ano. As demências constituem uma questão de saúde pública e trazem uma preocupação bioética com o envelhecimento: a perda da autonomia das pessoas afetadas e a responsabilidade do sistema de saúde para atendê-las.

Palavras-chave Demência, Envelhecimento, Demografia, Bioética 


\section{Introdução}

Uma das maiores conquistas das sociedades contemporâneas é o aumento da expectativa de vida. Este fato resulta da redução significativa das taxas de mortalidade por doenças infectocontagiosas e crônicas em todas as idades como consequência de melhorias das condições de vida em geral, do avanço da tecnologia médica e de um maior acesso a serviços de saúde ${ }^{1,2}$.

Em quase todo o mundo, o contingente que mais cresce é o de pessoas com idade igual ou superior a 60 anos. Segundo a Organização Mundial da Saúde (OMS) 3 754,6 milhões de indivíduos estavam nessa faixa etária em 2010. Trata-se da população nascida sob o regime de baby boom, isto é, num período de fecundidade muito alta e de mortalidade infantil declinante. Esse é o grupo que está entrando, atualmente, na coorte idosa: os baby boomers transformando-se nos elderly boomers.

Suas projeções apontam para 1,2 bilhão em 2025, podendo chegar aos 2 bilhões em 2050. Pela primeira vez na história da humanidade, as pessoas com 60 anos ou mais superarão as crianças menores de 14 anos, correspondendo, respectivamente, a $22,1 \%$ e $19,6 \%$ da população mundial.

A perspectiva para o Brasil é semelhante a essa, indicada pela OMS. Projeções demográficas $^{4}$ apontam para 2020 um contingente de 29,8 milhões de pessoas com 60 anos ou mais e de 4,7 milhões acima de 80 anos, significando, respectivamente, um acréscimo de 9,2 milhões e 1,7 milhão de indivíduos na faixa etária de 80 anos ou mais, comparado ao contingente de 2010.

No Brasil, os baby boomers são compostos pela geração nascida entre 1950 e 1965, grupo etário que estará com 55-70 anos em 2020. Essa geração, além de constituída por um volume demográfico expressivo, é responsável por grande parte das transformações vividas na contemporaneidade, por exemplo, nas áreas de comportamento social, sexualidade, formação familiar e relações de gênero. Tem como características uma grande preocupação com sua qualidade de vida e, acima de tudo, com a valorização da sua autonomia. Os baby boomers introduziram no âmbito sociopolítico a ideia de segurança social e preocupações com a ecologia, modificaram as relações familiares, o que teve um grande impacto social e apontaram para a necessidade de se preparar para a velhice, situação não experimentada por seus pais e seus avós $\mathrm{s}^{4,5}$.

Em outras palavras, por tais características, esses futuros idosos serão diferentes dos anteri- ores, especialmente as mulheres, porque elas estão vivenciando grandes ganhos na escolaridade e ocupam cada vez mais espaço no mercado de trabalho; promoveram a revolução sexual e a familiar e tiveram menos filhos, fatos que lhes trouxeram mais autonomia. Isso lhes traz independência financeira, mas também uma maior exposição ao estresse do mundo do trabalho e ao uso do álcool e do cigarro. Acredita-se que a exposição a estes fatores de risco afetará a sua saúde.

Camarano e Kanso ${ }^{5}$ mostraram que, ao longo do tempo, essa coorte de baby boomers continuou experimentando grandes reduções na mortalidade em várias fases de suas vidas. O resultado é uma coorte que nasceu numerosa e está desfrutando de grandes ganhos de sobrevivência. Em 2010, uma pessoa completando 60 anos de idade, se homem, poderia esperar viver mais 21,2 anos; e se mulher, 24,8. Estimativas da OMS (2004) de esperança de vida saudável apontam que os homens e as mulheres brasileiros de 60 anos e mais passam aproximadamente $40 \%$ do tempo vivido com alguma incapacidade. Isto representa 12 anos do tempo vivido pelos homens e 13 do vivido pelas mulheres ${ }^{6}$.

As autoras ${ }^{5}$ mostraram, também, que a mortalidade da população idosa brasileira pode ser ainda reduzida com a eliminação de mortes consideradas evitáveis, dado o atual estágio da tecnologia médica. Com base nessa estimativa, mulheres que atingissem 60 anos poderiam esperar viver mais 30,9 anos, ou seja, 6,1 anos a mais do que a expectativa estimada para 2010. Para os homens, as estimativas comparáveis são 29,2 e 8,0 anos, respectivamente. Acredita-se, porém, que a eliminação de mortes evitáveis trará uma convivência maior com morbidades não letais, entre elas as demências.

Viver mais implica no declínio fisiológico das funções orgânicas e, em razão disso, uma maior probabilidade de surgimento de doenças crônicas, incapacitantes e involutivas ${ }^{6}$, que podem comprometer a autonomia das pessoas. Um exemplo típico são as síndromes demenciais, cuja prevalência cresce com a idade, embora não seja um componente normal do envelhecimento. As demências consistem numa síndrome cujo efeito é acometer o funcionamento cerebral. Têm natureza crônica e progressiva, comprometendo várias funções cerebrais, incluindo memória, raciocínio, orientação, compreensão, cálculo, capacidade de aprendizagem, linguagem e julgamento ${ }^{7,8}$. Os déficits da função cognitiva são geralmente acompanhados e ocasionalmente pre- 
cedidos de uma deterioração do controle emocional, do comportamento social ou da motivação. A causa mais comum de demência é a Doença de Alzheimer (DA) respondendo por $60 \%$ a $70 \%$ dos casos, seguindo-se demência vascular, demência por corpos de Lewy e demência frontotemporal ${ }^{9,10}$.

Em abril de 2012, a OMS publicou o documento "Demência: Uma Questão de Saúde Pública" "11, demonstrando preocupação para esse problema que afeta a qualidade de vida das pessoas longevas, especialmente nos países em desenvolvimento. Estima-se em 35,6 milhões o número de pessoas com demência em 2010, projetando uma duplicação neste número a cada 20 anos; ou seja, 65,7 milhões em 2030 e 115,4 milhões em 2050. O total de casos novos de demência a cada ano no mundo é de aproximadamente 7,7 milhões, o que significa uma pessoa diagnosticada a cada quatro segundos.

A demência é uma das principais causas de incapacidade na velhice, demandando cuidados durante todo o curso desta enfermidade que culmina na dependência total da pessoa doente. De acordo com a $\mathrm{OMS}^{12}$, o tempo vivido com demência responde por $11,9 \%$ dos anos de convívio com incapacidades decorrentes de doenças não transmissíveis, apresentando, no ano de 2010, um custo mundial estimado em US\$ 604 bilhões ${ }^{13}$.

No Brasil, informações sobre incidência e prevalência de demências restringem-se a pequenas áreas geográficas e a determinados períodos de tempo, ou seja, não há continuidade e nem amplitude nos registros dessa doença. Na revisão de Nitrini et al. ${ }^{14}$ sobre a prevalência de demência em uma perspectiva global, a América Latina é considerada uma região na qual faltam informações de boa qualidade.

Este artigo tem como objetivo principal projetar o crescimento do número de idosos com demências no Brasil no futuro próximo. Parte-se do pressuposto de que o número de idosos com demências tende a crescer. Tomam-se por base estudos epidemiológicos que apresentavam informações sobre a incidência e a prevalência da demência na população idosa brasileira. Estas informações foram utilizadas para se obter um cenário do crescimento da população de idosos brasileiros que conviverão com demências nos próximos 10 anos.

\section{Procedimentos Metodológicos}

Dado ser o objetivo do trabalho a elaboração de projeções sobre o número de idosos demenciados, realizou-se uma estimativa do número atual, aplicando medidas de prevalência por idade à população de 65 anos e mais, desagregada por grupo de idade, enumerada pelo Censo Demográfico de 2010. Estas medidas foram obtidas de levantamentos detectados em uma revisão da literatura. Para se obter uma medida nacional, utilizou-se uma média ponderada das prevalências encontradas, sendo o peso o número de observações. Foram obtidas taxas de prevalência por idade e sexo para a população idosa brasileira.

Assumindo-se que tais prevalências não se alterariam ao longo desta década, elas foram aplicadas à população projetada por grupos de idade para 2020 por Camarano e Kanso ${ }^{4}$. Assim, chegou-se à projeção do total de idosos com demência em 2020.

A principal dificuldade para a elaboração de uma medida que projetasse a população idosa demenciada para um período futuro foi obter taxas de prevalência e de incidência de abrangência nacional. Para isto, buscou-se na literatura informações extraídas de estudos específicos, que continham informações para pequenas áreas. A revisão da literatura centrou-se em artigos referentes à prevalência e incidência de demência da população de idosos no Brasil. A busca foi feita nos bancos Medline, Lilacs e SCIELO, utilizando-se dos seguintes critérios de seleção:

1. Idiomas: português, inglês e espanhol;

2. Ano de publicação: de janeiro de 1995 a outubro de 2012;

3. Trabalhos referentes à população brasileira;

4. População alvo: indivíduos com 60 anos ou mais;

5. Artigos que continham explicitamente resultados numéricos de prevalência e/ou incidência.

A partir da leitura dos resumos e, quando necessário, da leitura completa do artigo foram selecionados aqueles com informações sobre prevalência e incidência no Brasil.

Embora o Estatuto do Idoso defina como população idosa a constituída por pessoas com idade igual ou superior a 60 anos, neste trabalho, considera-se idosa a população representada por pessoas de idade igual ou superior a 65 anos, porque a frequência de casos de demência no grupo de pessoas com idade de 60 a 64 anos é muito baixa. 


\section{Resultados}

Pela busca automatizada, onde foram definidas as palavras e os critérios de seleção, foram encontrados 703 estudos assim distribuídos: Medline (329), Lilacs (288) e SCIELO (86). Excluindo os estudos duplicados (447) restaram 256. Destes, 89 atendiam aos critérios de inclusão. Por meio de uma análise mais refinada, à luz dos objetivos desta pesquisa e dos critérios de inclusão, foram selecionados 13 estudos, sendo dez sobre prevalência e três sobre incidência.

Todas as medidas se referem a pequenas áreas do território nacional, a maioria localizada no estado de São Paulo. Dos estudos de prevalência, dois se referem às mesmas pesquisas, ou seja, apresentam dados duplicados. O resultado final foi de oito medidas, que estão apresentadas na Tabela 1, desagregadas por grupos de idade e pela pesquisa a que se referem. Apenas sete estudos apresentaram dados por sexo, o que pode ser visto na Tabela 2. Encontra-se, também, na Tabela 1 a prevalência média para a América Latina e a mundial.

A taxa bruta de prevalência variou de 2,03\% em Piraju a $12,10 \%$ na comunidade japonesa em Campo Grande. A média observada para os países da América Latina foi de 7,13\%. Diferenças expressivas também foram observadas nas taxas por grupos de idade. Uma característica observada nos oito estudos, na média mundial e da América Latina é que a prevalência de demência cresce acentuadamente com a idade, variando de $0,12 \%$ na faixa etária de 65 a 69 anos em Piraju (SP) a 77,8\% entre os idosos de 90 a 94 anos da comunidade japonesa de Campo Grande.

Outra variável observada foi sexo. Entre os homens, as taxas de prevalência variaram de 4,2\% no Distrito de Butantã em São Paulo a 11,4\% na comunidade japonesa de Campo Grande. Para as mulheres, essa variação foi mais expressiva, de $2,2 \%$ em Piraju (SP) a $12,6 \%$ na comunidade japonesa de Campo Grande. Em todas as áreas estudadas a taxa de prevalência foi maior entre as mulheres. Os maiores diferenciais por sexo foram observados em Catanduva e em uma comunidade japonesa de São Paulo. As taxas para as mulheres foram $81 \%$ e $42 \%$ maior que a dos homens, respectivamente.

Dos estudos encontrados, apenas três apresentavam também medidas de incidência de demências. O primeiro se refere a idosos residentes no município de Catanduva ${ }^{15}$. Estas foram obtidas de um grupo de 1.538 indivíduos de 65 anos diagnosticados com demência e observados em
2000. A incidência da demência encontrada foi de 13,8 por 1.000 pessoas/ano, sendo a da DA de 7,7 por 1.000. O segundo estudo, de Chaves et al. ${ }^{16}$, avaliou a incidência de casos da DA e de comprometimento cognitivo leve em uma coorte de 345 idosos da comunidade do Hospital de Clínicas de Porto Alegre, encontrando taxas de 14,8 por 1.000 idosos e 13,2 por 1.000, respectivamente. No estudo de Catanduva, desenvolvido por Nitrini et al. ${ }^{15}$, as taxas de incidência foram estratificadas por idade, sexo, escolaridade e nível socioeconômico e os resultados vão no mesmo sentido dos obtidos nas medidas de prevalência.O terceiro artigo, de autoria de Meguro et al. ${ }^{17}$, aborda a incidência de demência em idosos imigrantes japoneses no Brasil e apresenta resultados da segunda etapa do estudo iniciado em 1997 sobre a prevalência de demência entre esses idosos que viviam na área metropolitana de São Paulo. Esta segunda etapa foi realizada em 2009, com o objetivo de estudar as causas de morte e a incidência de demência. Do total de idosos japoneses que participaram da pesquisa na primeira etapa, 54 faleceram antes da realização da segunda, restando 108 indivíduos. Entre os falecidos, cerca de $30 \%$ haviam desenvolvido demência e 20,8\% dos indivíduos vivos haviam sido diagnosticados com demência. A taxa de incidência foi de 34,2 por 1.000 pessoas/ano.

Quanto ao tipo de demência, no estudo de Catanduva $^{18}$, os autores encontraram Doença de Alzheimer em 54,1\% dos casos analisados, demência vascular em 9,4\% e demência mista em $14,5 \%$. Resultados semelhantes foram observados por Meguro et al. ${ }^{17,19}$.

Com base nas medidas de prevalência encontradas, estimou-se a medida de prevalência da demência na população idosa brasileira. Os resultados constam da Tabela 3. A taxa de prevalência foi calculada em 7,6\%.

A projeção do número de demenciados resultou em um contingente de aproximadamente 1.069 mil idosos brasileiros em 2010. Mantendose constantes as taxas de prevalência ao longo desta década, pode-se esperar encontrar 1.633 mil idosos nessa condição em 2020. Este cenário implicaria em um aumento de, aproximadamente, 560 mil idosos demenciados, ou seja, de 53\%, resultado apenas de fatores demográficos, isto é, do esperado crescimento da população idosa. A taxa de prevalência geral aumentaria ligeiramente pelo envelhecimento populacional. Passaria de 7,6\% para 7,9\%. Em termos de incidência, seriam 2,7 novos idosos demenciados em cada 1.000 idosos a cada ano. 
Tabela 1. Prevalência de demência por idade (Brasil).

\begin{tabular}{|c|c|c|c|c|}
\hline \multirow[b]{2}{*}{ Pesquisa } & \multicolumn{4}{|c|}{ Idade } \\
\hline & \multicolumn{2}{|r|}{$70-74$} & $75-79$ & $80-84$ \\
\hline $\begin{array}{l}\text { Catanduva (SP) - } 1996(1) \\
\mathrm{N}=1.656\end{array}$ & 1,63 & 3,19 & 7,89 & 15,15 \\
\hline $\begin{array}{l}\text { Piraju (SP) }- \text { sd (2) } \\
\mathrm{N}=2.222\end{array}$ & 0,12 & 1,23 & 2,59 & 3,13 \\
\hline $\begin{array}{l}\text { São Paulo (SP) - 2002/2003 (3) } \\
\mathrm{N}=1.563\end{array}$ & 4,10 & 7,10 & 9,50 & 13,28 \\
\hline $\begin{array}{l}\text { Distrito de Butantã (SP) - 2003/2005 (4) } \\
\mathrm{N}=2.072\end{array}$ & $\begin{array}{c}2,30 \\
(1,3-3,2)\end{array}$ & 2,00 & $\begin{array}{c}7,80 \\
(5,0-10,6)\end{array}$ & $\begin{array}{c}13,60 \\
(8,4-18,8)\end{array}$ \\
\hline $\begin{array}{l}\text { Comunidade japonesa (SP) }- \text { sd (5) } \\
\mathrm{N}=166\end{array}$ & 0,00 & 2,20 & 4,70 & 6,30 \\
\hline $\begin{array}{l}\text { Comunidade japonesa/Campo Grande } \\
\text { (MS) - } 2000(6) \\
\mathrm{N}=157\end{array}$ & \multicolumn{2}{|r|}{1,60} & 6,50 & 16,70 \\
\hline $\begin{array}{l}\text { Ribeirão Preto }- \text { sd }(7)^{*} \\
N=1.145 \\
\text { Santa Cruz }(\mathrm{RN})-2009(10) \\
\mathrm{N}=59\end{array}$ & 2,21 & 5,58 & 8,82 & 12,75 \\
\hline América Latina (8) & $\begin{array}{c}2,40 \\
(2,11-2,72)\end{array}$ & $\begin{array}{c}3,57 \\
(3,18-4,00)\end{array}$ & $\begin{array}{c}7,04 \\
(6,41-7,69)\end{array}$ & $\begin{array}{c}11,88 \\
(10,87-12,91)\end{array}$ \\
\hline Média Mundial - 1994 a 1999 (9) & \multicolumn{2}{|l|}{$\begin{array}{c}1,17 \\
(0,70-1,63)\end{array}$} & $\begin{array}{c}7,87 \\
\text { 36) } \quad(6,10-9,64)\end{array}$ & $\begin{array}{c}16,22 \\
(12,49-19,95)\end{array}$ \\
\hline & \multicolumn{4}{|c|}{ Idade } \\
\hline Pesquisa & 85-89 & $90-94$ & Taxa Bruta Tax & ra Padronizada \\
\hline $\begin{array}{l}\text { Catanduva (SP) - } 1996 \text { (1) } \\
\mathrm{N}=1.656\end{array}$ & 38,90 & & $\begin{array}{c}7,13 \\
(6,0-8,5)\end{array}$ & \\
\hline $\begin{array}{l}\text { Piraju (SP) }- \text { sd (2) } \\
\mathrm{N}=2.222\end{array}$ & 12,05 & & 2,03 & \\
\hline $\begin{array}{l}\text { São Paulo (SP) - 2002/2003 (3) } \\
\mathrm{N}=1.563\end{array}$ & 15,28 & 42,31 & $\begin{array}{c}8,30 \\
(6,7-9,9)\end{array}$ & 6,60 \\
\hline $\begin{array}{l}\text { Distrito de Butantã (SP) - 2003/2005 (4) } \\
\mathrm{N}=2.072\end{array}$ & $\begin{array}{c}21,40 \\
(13,7-29,1)\end{array}$ & & $\begin{array}{c}5,10 \\
(4,1-6,0)\end{array}$ & $(5,4-7,8)$ \\
\hline $\begin{array}{l}\text { Comunidade japonesa (SP) }- \text { sd (5) } \\
\mathrm{N}=166\end{array}$ & $28,6^{* *}$ & & 7,80 & \\
\hline $\begin{array}{l}\text { Comunidade japonesa/Campo Grande } \\
\text { (MS) - } 2000(6) \\
\mathrm{N}=157\end{array}$ & 26,70 & 77,80 & $12,1^{* * *}$ & \\
\hline $\begin{array}{l}\text { Ribeirão Preto }- \text { sd }(7)^{*} \\
N=1.145 \\
\text { Santa Cruz }(\mathrm{RN})-2009(10)\end{array}$ & 15,00 & 38,10 & $\begin{array}{c}7,24 \\
(5,7-8,6) \\
11,8^{* * * *}\end{array}$ & \\
\hline $\mathrm{N}=59$ & & & & 5,97 \\
\hline América Latina (8) & $\begin{array}{c}20,20 \\
(18,62-21,78)\end{array}$ & $\begin{array}{c}33,07 \\
(29,98-36,20)\end{array}$ & $\begin{array}{c}7,13 \\
(6,86-7,42)\end{array}$ & $(5,91-6,06)$ \\
\hline Média Mundial - 1994 a 1999 (9) & $\begin{array}{c}23,5 \\
(17,63-29,37) \quad(\end{array}$ & $\begin{array}{c}38,76 \\
(31,75-45,76)\end{array}$ & & \\
\hline
\end{tabular}

${ }^{*}$ Cálculo elaborado pelos autores. ${ }^{* *} \mathrm{O}$ grupo de $85-89$ foi o último grupo de idade pesquisado. ${ }^{* *}$ Refere-se ao grupo de 70 anos ou mais de idade. ${ }^{* * *}$ Valores do teste LCT, para o exame MME o valor é 37,2\%. Os grupos de idade foram 65-69 e 70-74.

Fontes: 1 - Herrera Júnior et al. ${ }^{20} ; 2$ - Ramos-Cerqueira et al. ${ }^{21} ; 3$ - Bottino et al. ${ }^{22} ; 4$ - Scazufca et al..$^{23} ; 5$ - Meguro et al. ${ }^{19} ; 6$ Yamada et al. ${ }^{24} ; 7$ - Lopes $^{25} ; 8$ - Nitrini et al. ${ }^{14} ; 9$ - Lopes e Bottino ${ }^{26} ; 10$ - Caldas et al. ${ }^{27}$. 
Tabela 2. Prevalência de demência por sexo (Brasil)

\begin{tabular}{lccc}
\hline \multicolumn{1}{c}{ Pesquisa } & \multicolumn{2}{c}{ Sexo } & \multirow{2}{*}{ Total } \\
\cline { 2 - 3 } & Homens & Mulheres & \\
\hline Catanduva (SP) - 1996 (1) & 5,2 & 9,4 & 7,1 \\
Piraju (SP) - sd (2) & 1,7 & 2,2 & 2,0 \\
São Paulo (SP) - 2002/2003 (3) & 7,1 & 7,3 & 8,3 \\
Distrito de Butantã (SP) - 2003/2005 (4) & 4,5 & 5,4 & 5,1 \\
Comunidade japonesa (SP) - sd (5) & 6,4 & 9,1 & 7,8 \\
Comunidade japonesa/Campo Grande (MS) - 2000 (6) & 11,4 & 12,6 & 12,1 \\
Ribeirão Preto (SP) - sd (7)* & 5,97 & 6,06 & 7,2 \\
\hline
\end{tabular}

* Cálculo elaborado pelos autores.

Fontes: 1 - Herrera Júnior et al. ${ }^{20} ; 2$ - Ramos-Cerqueira et al. ${ }^{21} ; 3$ - Bottino et al. ${ }^{22} ; 4$ - Scazufca et al..$^{23} ; 5$ - Meguro et al. ${ }^{19} ; 6$ Yamada et al. ${ }^{24} ; 7$ - Lopes ${ }^{25} ; 8$ - Nitrini et al. ${ }^{14} ; 9$ - Lopes e Bottino ${ }^{26} ; 10$ - Caldas et al. ${ }^{27}$.

Tabela 3. Simulações sobre prevalência de demência (Brasil).

\begin{tabular}{lccc}
\hline \multirow{2}{*}{$\begin{array}{c}\text { Grupos } \\
\text { de Idade }\end{array}$} & $\begin{array}{c}\text { Prevalência } \\
(\%)\end{array}$ & \multicolumn{2}{c}{$\begin{array}{c}\text { Demenciados } \\
\text { (por } \mathbf{1 . 0 0 0} \text { idosos) }\end{array}$} \\
\cline { 3 - 4 } & & $\mathbf{2 0 1 0}$ & $\mathbf{2 0 2 0}$ \\
\hline $65-69$ & 1,89 & 91,52 & 135,24 \\
$70-74$ & 3,34 & 125,02 & 175,94 \\
$75-79$ & 6,76 & 173,30 & 238,47 \\
80 e mais & 23,13 & 679,05 & $1.083,06$ \\
Total & $\mathbf{7 , 5 9}$ & $\mathbf{1 . 0 6 8 , 8 9}$ & $\mathbf{1 . 6 3 2 , 7 1}$ \\
& & & \\
\hline
\end{tabular}

Fonte: Estimativas das autoras

\section{Discussão dos resultados}

Apesar das taxas de prevalência apresentadas pela literatura referirem-se a apenas oito pequenas áreas brasileiras, pode-se observar grandes diferenças entre elas, o que reflete diferenças no diagnóstico, nos métodos e nos processos de coleta de dados de cada estudo, que ocorreram em diferentes momentos. Diferenças regionais nas prevalências também foram observadas nos inquéritos populacionais realizados em países desenvolvidos, assim como nos brasileiros ${ }^{14}$.

Com exceção do estudo de Piraju, os demais encontraram uma prevalência mais elevada que a média mundial para todas as idades, conforme mostrou a Tabela 1 . Nitrini et al. ${ }^{14}$, com base em uma revisão bibliográfica sobre a América Latina, verificaram que as taxas de prevalência de idosos dos seis países estudados, a saber, Brasil,
Chile, Cuba, Peru, Uruguai e Venezuela apresentaram-se mais elevadas do que as encontradas na maioria dos estudos e nas meta-análises realizadas em países desenvolvidos, como por exemplo Canadá e Estados Unidos.

Vários estudos, como por exemplo os de Lopes $^{25}$, Lopes e Bottino ${ }^{26}$ e de Nitrini et al. ${ }^{15}$, mostram que as mulheres têm uma prevalência de demência mais elevada que os homens, o que é coerente com o fato de elas conviverem por um período mais longo com algum tipo de incapacidade. As diferenças entre homens e mulheres nessas prevalências variam regionalmente, o que pode ser explicado pela heterogeneidade do perfil etário pelo território nacional. As maiores diferenças foram observadas em Catanduva ${ }^{18}$ e em uma comunidade japonesa de São Paulo ${ }^{17}$. No entanto, apesar de as mulheres registrarem proporções mais elevadas do que os homens, as pesquisas aqui referidas, de Lopes ${ }^{25}$, Herrera Júnior et al. ${ }^{18}$, Nitrini et al. ${ }^{15}$, Meguro et al. ${ }^{17,19}$ e Lopes e Bottino $^{26}$ concluíram que as diferenças por sexo não eram estatisticamente significantes. Por isso, não se infere que ser do sexo feminino implica em maior chance de surgimento de demências. São necessárias novas pesquisas para que se possa chegar a uma conclusão efetiva.

Estudos sobre a incidência da demência são menos frequentes tanto na literatura nacional quanto internacional. Os custos de seu levantamento são altos, pois para a identificação de novos casos é necessário o acompanhamento longitudinal dos indivíduos. No entanto, assim como os estudos de prevalência, aqueles também podem contribuir para a avaliação da carga da doença, para o planejamento de políticas de 
saúde e para a investigação dos respectivos fatores de risco ${ }^{15}$. Medidas de incidências são mais precisas para se avaliar a evolução da doença.

À semelhança do observado nas taxas de prevalência, as maiores taxas de incidência foram encontradas entre os mais idosos, menos escolarizados e com níveis socioeconômicos mais baixos. A comparação entre homens e mulheres apontou que, até os 84 anos, as mulheres registraram as maiores taxas. Foram encontradas grandes diferenças entre as medidas de Catanduva e de Porto Alegre. Esta diferença pode ser resultado de variações de diagnóstico e do elevado número de pessoas hospitalizadas com doenças crônicas, caso de Porto Alegre.

Os resultados da estimativa do número de idosos demenciados apontou um valor semelhante às estimativas da $\mathrm{OMS}^{11}$ para 2010, $1.069 \mathrm{mil}$ idosos e um milhão de idosos brasileiros demenciados, respectivamente. A taxa de prevalência foi calculada em 7,6\%, valor semelhante ao observado para a média dos países da América Latina, $7,1 \%$, conforme mostrado por Nitrini et al. ${ }^{14}(\mathrm{Ta}-$ bela 2). A medida de incidência encontrada foi mais baixa que a estimada por Nitrini et al. ${ }^{15}, 2,7$ por 1000 e 13,8 por 1.000 , respectivamente. Isto pode, em parte, ser explicado pelas simulações aqui realizadas utilizarem apenas as medidas de prevalência e o crescimento demográfico.

\section{Considerações Finais}

O objetivo deste trabalho foi a obtenção de uma estimativa do crescimento de idosos demenciados no futuro próximo. Espera-se, com isso, subsidiar o processo de formulação de políticas na área de saúde pública. Os resultados apontaram para um crescimento desse segmento, dado o aumento esperado da população muito idosa, ainda que a prevalência não cresça. Chamam a atenção para uma nova e urgente questão ética: o cuidado necessário a ser dispensado às pessoas idosas com demência.

Chamam, também, a atenção para o fato de os dados apresentados constituírem apenas uma aproximação de uma medida de prevalência de demência na população idosa brasileira dada a carência de informações para todo o território nacional. Qualquer proposta de política para atender esse segmento populacional, no Brasil, esbarra na carência de informações sobre a prevalência e a incidência dessa doença.

Seguindo o aforismo que sintetiza a Medicina e o compromisso do médico de "curar algu- mas vezes, aliviar muitas vezes e consolar sempre”, em relação às demências, é imperativo o desenvolvimento da consciência da prevenção quando possível; a criação de solução muitas vezes e a sempre promoção do alívio do sofrimento causado por essa doença. Essa consciência envolve os serviços e as políticas para a área da saúde e todos os profissionais incumbidos do tratamento e do acompanhamento das pessoas demenciadas.

A tensão entre a realidade demográfica e a epidemiológica, a biotecnologia, a disponibilidade de recursos em saúde e o cuidado necessário às pessoas idosas com demência levantam questões éticas inadiáveis. A proposta da Bioética, ressaltando a complexidade da autonomia de decisão e a radical demanda de proteção no cenário das demências, longe de um apaziguamento com ofertas de soluções, é um desafio. Este aponta inquietações das quais devem surgir respostas condizentes com os valores morais da sociedade e com o direito fundamental de preservação da dignidade em qualquer circunstância, condição inalienável do ser humano. Novos estudos serão necessários para determinar em que medida é que pode ser efetuada a deteção precoce de estados demenciais, permitindo assim que cada pessoa possa efetuar escolhas de acordo com os seus referenciais de valores, definindo através da elaboração de uma diretiva antecipada de vontade, seja pela redação de um testamento vital ou da nomeação de um procurador de cuidados de saúde.

\section{Colaboradores}

C Burlá e AA Camarano trabalharam na elaboração do texto, concepção, metodologia e redação final. S Kanso e D Fernandes trabalharam na pesquisa. R Nunes trabalhou na revisão final.

\section{Agradecimentos}

À Prof. Claudia Travassos pela leitura cuidadosa de versões anteriores a este trabalho e os valiosos comentários daí advindos. 


\section{Referências}

1. Kalache A, Veras RP, Ramos LR. The ageing of the world population: a new challenge. Rev Saude Publica 1987; 21(3):200-210.

2. Chaimowicz F. Envelhecimento e Saúde no Brasil. In: Freitas EV, Py L, organizadores. Tratado de geriatria e gerontologia. Rio de Janeiro: Guanabara-Koogan; 2011. p. 74-98.

3. World Health Organization. Ageing and life course [homepage na internet]. 2008 [acessado 2012 ago 13]. Disponível em: http://www.who.int/ageing/en/

4. Camarano AA, Kanso S. Perspectivas de crescimento para a população brasileira: velhos e novos resultados. Rio de Janeiro: Ipea; 2009. (Texto para Discussão, n. 1.426).

5. Camarano AA, Kanso S. Envelhecimento da população brasileira: uma contribuição demográfica. In: Freitas EV, Py L, organizadores. Tratado de geriatria e gerontologia. Rio de Janeiro: Guanabara-Koogan; 2011. p. 58-73.

6. Jeckel-Neto EA, Cunha GL. Teorias biológicas do envelhecimento. In: Freitas EV, Py L, Neri A L, Cançado FAX, Gorzoni ML, Doll J, organizadores. Tratado de geriatria e gerontologia. Rio de Janeiro: Guanabara-Koogan; 2006. p. 13-22.

7. American Psychiatric Association. Diagnostic and Statistical Manual of Mental Disorders. $4^{\text {th }}$ Edition. Washington DC: American Psychiatric Association; 1994.

8. Starr JM. The older adult with intellectual disability. In: Fillit HM, Rockwood K, Woodhouse K, organizadores. Brocklehurst's Textbook of geriatric medicine and gerontology. $7^{\text {th }}$ Edition. Philadelphia: Saunders, Elservier; 2010. p. 445-452.

9. World Alzheimer's Report 2009. London: Alzheimer's Disease International; 2009.

10. Farlow MR. Alzheimer disease. In: Fillit HM, Rockwood K, Woodhouse K, organizadores. Brocklehurst's Textbook of geriatric medicine and gerontology. $7^{\text {th }}$ Edition. Philadelphia: Saunders, Elservier; 2010. p. 411-420.

11. World Health Organization (WHO). Dementia: a public health priority. Geneva: WHO; 2012.

12. World Health Organization (WHO). The global burden of disease: 2004 update. Geneva: WHO; 2008.

13. World Alzheimer's Report 2010: the global economic impact of dementia. London: Alzheimer's Disease International; 2010.

14. Nitrini R, Bottino CMC, Albala C, Capuñay NSC, Ketzoian C, Juan J. Prevalence of dementia in Latin America: a collaborative study of population-based cohorts. Int Psychogeriatr 2009; 21(4):622-630.

15. Nitrini R, Caramelli P, Herrera E, Bahia VS, Caixeta LF, Radanovic M, Anghinah R, Charchat-Fichman H, Porto CS, Carthery MT, Hartmann APJ, Huang N, Smid J, Lima EP, Takada LT, Takahashi DY. Incidence of Dementia in a Community-Dwelling Brazilian Population. Alzheimer Dis Assoc Disord 2004; 18(4):241-246.

16. Chaves ML, Camozzato AL, Godinho C, Piazenski I, Kaye J. Incidence of Mild Cognitive Impairment and Alzheimer Disease in Southern Brazil. J Geriatr Psychiatry Neurol 2009; 22(3):181-187.
17. Meguro K, Chubaci RYS, Meguro M, Kawamorida $\mathrm{K}$, Goto N, Caramelli P. Incidence of dementia and cause of death in elderly Japanese emigrants to Brazil before World War II. Arch Gerontol Geriatr 2011; 52(1):75-78.

18. Herrera Júnior E, Caramelli P, Nitrini R. Estudo epidemiológico populacional de demência na cidade de Catanduva - estado de São Paulo - Brasil. Rev Psiquiatr Clin 1998; 25(2):70-73.

19. Meguro K, Meguro M, Caramelli P, Ishizaki J, Ambo H, Chubaci RY, Hamada GS, Nitrini R, Yamadori A. Elderly Japanese emigrants to Brazil before World War II: II. Prevalence of senile dementia. Int J Geriatr Psychiatry 2001; 16(8):775-779.

20. Herrera Júnior E, Caramelli P, Silveira AS, Nitrini R. Epidemiologic Survey of Dementia in a Community-Dwelling Brazilian Population. Alzheimer Dis Assoc Disord 2002; 16(2):103-108.

21. Ramos-Cerqueira AT, Torres AR, Crepaldi AL, Oliveira NIL, Scazufca M, Menezes PR, Prince M. Identification of Dementia Cases in the Community: A Brazilian Experience. J Am Geriatr Soc 2005; 53(10):1738-1742.

22. Bottino CM, Azevedo DJ, Tatsch M, Hototian SR, Moscoso MA, Folquitto J, Scalco AZ, Bazzarella MC, Lopes MA, Litvoc J. Estimate of Dementia Prevalence in a Community Sample from São Paulo, Brazil. Dement Geriatr Cogn Disord 2008; 26(4):291-299.

23. Scazufca M, Menezes PR, Vallada HP, Crepaldi AL, Pastor-Valero M, Coutinho LM, Di Rienzo VD, Almeida OP. High prevalence of dementia among older adults from poor socioeconomic backgrounds in São Paulo, Brazil. Int Psychogeriatr 2008; 20(2): 394-405.

24. Yamada T, Kadekaru H, Matsumoto S, Inada H, Tanabe M, Moriguchi EH, Moriguchi Y, Ishikawa P, Ishikawa AG, Taira K, Yamori Y. Prevalence of dementia in the older Japanese-Brazilian population. Psychiatry Clin Neurosci 2002; 56(1):71-75.

25. Lopes MA. Estudo epidemiológico de prevalência de demência em Ribeirão Preto [tese]. São Paulo: Faculdade de Medicina da Universidade de São Paulo; 2006.

26. Lopes MA, Bottino CM. Prevalência de demência em diversas regiões do mundo: Análise dos estudos epidemiológicos de 1994 a 2000. Arq Neuropsiquiatr 2002; 60(1):61-69.

27. Caldas VVA, Zunzunegui MV, Freire ANF, Guerra RO. Translation, cultural adaptation and psychometric evaluation of the Leganés cognitive test in a low educated elderly Brazilian population. Arq Neuropsiquiatr 2012; 70(1):22-27.

Artigo apresentado em 14/11/2012

Aprovado em 30/11/2012

Versão final apresentada em 02/12/2012 